\title{
HIDRODINÂMICA E DISTRIBUIÇÃO DE SÓLIDOS EM SUSPENSÃO NO ESTUÁRIO DO RIO ITAJAÍ-AÇU. [RELATÓRIO]*
}

\author{
SCHETTINI, C.A.F. \& J.L.B. CARVALHO \\ CTTMar/UNIVALI
}

\begin{abstract}
RESUMO
Este relatório apresenta os resultados obtidos durante as campanhas de coleta de dados hidrográficos realizadas no estuário do Rio Itajaí-açu, em novembro de 1994 e em junho de 1995. Estas campanhas consistiram de experimentos de 25 horas de duração com aquisição de dados de correntes (velocidade e direção), salinidade e turbidez, a cada hora e em duas estações sinópticas. As informações são fornecidas na forma de gráficos com a distribuição do campo dos parâmetros coletados, na forma de dados reduzidos, e como valores médios dos parâmetros e a variação máxima de cada parâmetro observado.
\end{abstract}

Palavras chave: (1) Rio Itajaí-açu; (2) estuário; (3) hidrodinâmica; (4) material em suspensão.

\section{HYDRODYNAMICS AND SUSPENDED SEDIMENT DISTRIBUTION OF THE ITAJAÍ-AÇU RIVER ESTUARY. [TECHNICAL REPORT]}

\begin{abstract}
This report presents the results of the hydrographic surveys in the Itajaí-açu River estuary carried out during November, 1994 and June, 1995. These surveys consisted of 25 hours experiments to record currents, salinity and turbidity hourly in two sampling stations sinoptically. The information is presented as field distribution charts of the recorded parameters, in post-processed data tables, with information to surface, middle-depth and near bottom levels, and also as averaged values for each level and station, and maximum range as well.
\end{abstract}

Keywords: (1) Itajaí-açu river; (2) estuary; (3) hydrodynamic; (4) suspended sediments.

\section{INTRODUÇÃO}

A linha de costa do Estado de Santa Catarina é privilegiada em relação à riqueza de feições geomorfológicas e ambientes aquáticos. A proximidade do embasamento rochoso da linha de costa permite a presença de inúmeros promontórios que formam baías e enseadas, e.g., Baía de Babitonga, Enseada da Armação do Itapocoroy, etc... Lagoas costeiras e rios mareais também são observados ao longo do litoral nas regiões com planícies costeiras mais extensas, e.g., Lagoa do Imaruí, Rio Itajaí-açu.

A avaliação dos processos oceanográficos que se desenrolam nestes ambientes é de fundamental importância para a compreensão dos fatores que controlam a qualidade da água na região costeira. Apesar da importância destes ambientes e da qualidade da

*Trabalho realizado com recursos da Fundação Banco do Brasil. 
água costeira para a economia regional (turismo), poucos estudos que permitam o seu gerenciamento racional foram realizados até o momento.

O objetivo do presente trabalho é de divulgar os resultados obtidos através do "Projeto Integrado da Foz do Rio Itajaí-açu e Região Costeira Adjacente: Diagnóstico e Avaliação Ambiental', o qual possibilitou aprofundar os conhecimentos sobre os processos estuarinos no Rio Itajaí-açu e também adquirir as primeiras informações sobre a hidrodinâmica dos estuário dos rios Itapocu, Tijucas e Camboriu (Schettini \& Carvalho, neste volume).

O presente trabalho descreve os resultados de levantamentos de dados hidrográficos e sedimentológicos realizados no estuário do Rio Itajaí em 1994 e 1995. Diversas publicações já foram realizadas utilizando as informações adquiridas por este projeto (Schettini \& Carvalho, 1996; Schettini et al., 1996; Schettini et al., 1997; Schettini \& CarvaIho, 1998), sendo que este trabalho objetiva disponibilizar os dados obtidos em uma forma reduzida, permitindo assim o seu uso por outros pesquisadores.

\section{CARACTERIZAÇÃO GERAL}

O estuário do Rio Itajaí-açu é o maior contribuinte de descarga fluvial para o litoral de Santa Catarina, apresentando uma descarga média de $318 \pm 394 \mathrm{~m}^{3} . \mathrm{s}^{-1}$, calculada com dados diários de 1934 até 1997 fornecidos pela Agência Nacional de Energia Elétrica. O Rio Itajaí-açu drena um vale de $15.500 \mathrm{~km}^{2}$, onde está localizado o principal parque industrial do estado, com as cidades de Rio do Sul, Blumenau, Brusque e Itajaí (Figura 1). Próximo à desembocadura está localizado o Porto de Itajaí, sendo a principal via de comércio internacional do Estado, e uma das mais importantes do Brasil.

Apesar de sua importância estratégica, poucos estudos foram realizados até o mo- mento sobre as características hidrodinâmicas deste estuário. Os poucos estudos que existem estão concentrados na porção próxima à desembocadura, na região de interesse do porto. Ponçano $(1982,1987)$ caracterizou a distribuição faciológica do canal de acesso ao porto, verificando uma grande variabilidade em função da descarga fluvial. Döbereiner (1985) verificou que a circulação predominante no trecho estuarino é do tipo altamente estratificada, segundo os padrões de Pritchard (1955), sendo que a penetração da cunha salina varia em função das condições de descarga fluvial. Em condições de descarga em torno de $200 \mathrm{~m}^{3} . \mathrm{s}$ 1 , a penetração da cunha salina alcança aproximadamente $20 \mathrm{~km}$ à montante da barra, $\mathrm{e}$ durante períodos que a descarga excede valores de $1.000 \mathrm{~m}^{3} \cdot \mathrm{s}^{-1}$, a cunha salina é totalmente expulsa da bacia estuarina.

Estes resultados indicam que o estuário do Rio Itajaí-açu é regulado principalmente pelas condições fluviais. Em função da gran-

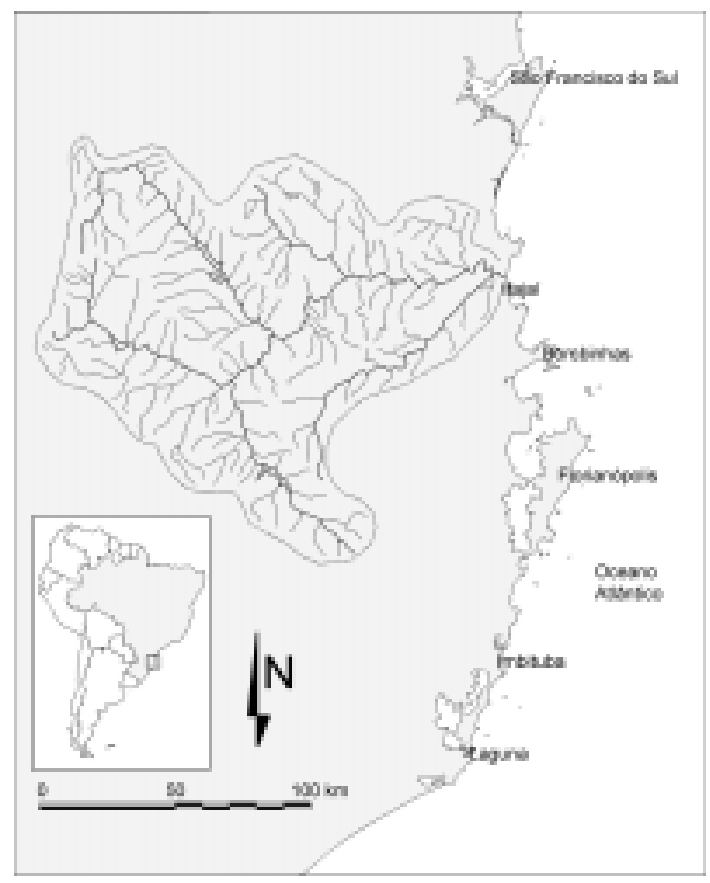

Figura 1: Localização da bacia de drenagem do Rio Itajaíaçu, no contexto da linha de costa de Santa Catarina. 
de variabilidade temporal que a descarga fluvial apresenta em resposta aos fenômenos meteorológicos, os processos de circulação estuarinos respondem com uma variabilidade ainda maior devido a adição dos efeitos das marés e dos ventos. Considerando isto, a metodologia adotada para avaliar com mais detalhe a hidrodinâmica deste ambiente foi a de experimentos de curta duração com alta freqüência amostral.

\section{MATERIAL \& MÉTODOS}

Foram realizados três experimentos de 25 horas de duração, equivalente a dois ciclos completos de maré. Os experimento foram realizados em: (1) Campanha \# 1: 3 e 4 de novembro de 1994, durante maré de sizígia; (2) Campanha \# 2: 6 e 7 de junho de 1995, durante maré de quadratura; e (3) Campanha \# 3: 13 e 14 de junho de 1995, durante maré de sizígia. As informações foram coletadas em duas estações sinópticas, sendo a Estação \# 1 localizada próxima da barra (» $8 \mathrm{~m}$ de profundidade) e a Estação \# 2 localizada 12 km à montante (» $7 \mathrm{~m}$ de profundidade; Figura 2).

I-Dados de nível da água

Dados de nível forma coletados com réguas graduadas fixas nas margens, em intervalos quase regulares inferiores à 30 minutos.

\section{II-Dados de corrente}

Na Campanha \# 1 foram coletados dados de velocidade e direção de corrente próximo da superfície (»1 $\mathrm{m}$ abaixo) e próximo do fundo (» $1 \mathrm{~m}$ acima) com o uso de correntógrafos SensorDataTM SD6000. Nas Campanhas \# 2 e \# 3, foi utilizado um correntômetro Consub ${ }^{\mathrm{TM}}$ na Estação \#2, o que permitiu realizar medições de velocidade e direção de corrente a cada metro de coluna de água.

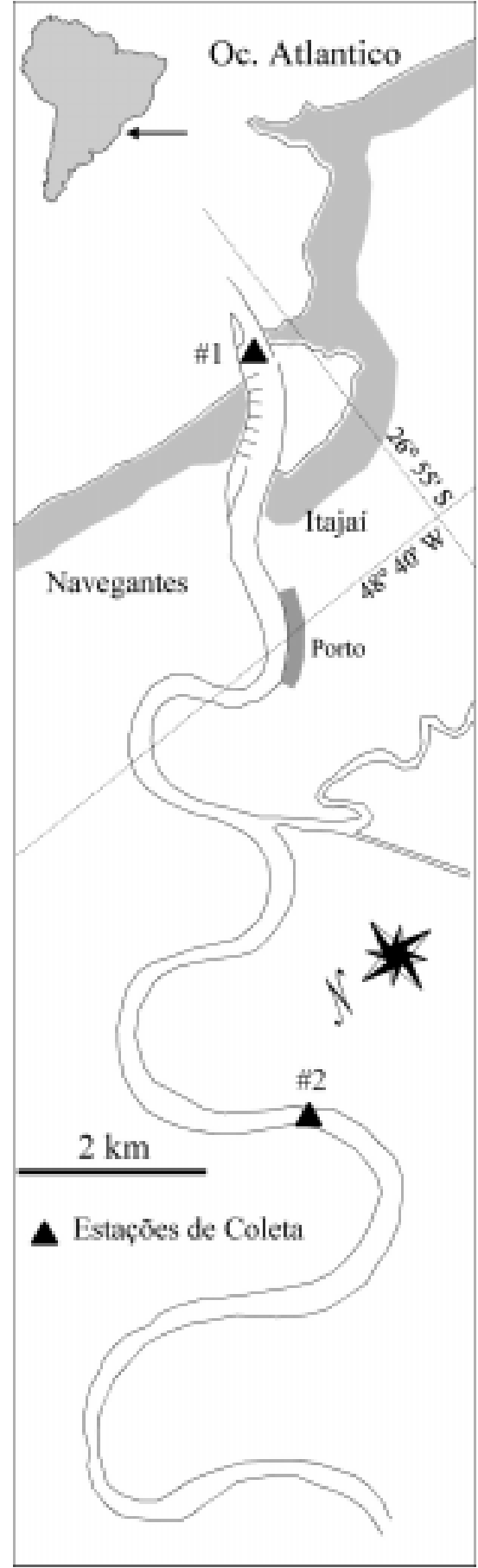

Figura 2: Localização das estações de amostragem no Estuário do Rio Itajaí-açu. 


\section{III - Dados de salinidade}

Na Campanha \# 1 amostras de água foram coletadas na Estação \# 2 na superfície, à meia água e próximas do fundo para determinação da salinidade em laboratório com o uso de um condutivímetro. Na Estação \# 1 foi utilizado uma sonda multiparâmetro Horiba ${ }^{\mathrm{TM}}$, sendo realizadas leituras de salinidade a cada metro de coluna de água. Nas Campanhas \# 2 e \# 3, foram utilizadas sondas Horiba ${ }^{\mathrm{TM}}$ em ambas as estações.

\section{IV - Sedimentos em suspensão}

Amostras de água para determinação de sedimentos em suspensão foram coletadas nas Campanhas \# 2 e \# 3, na superfície, meia água e próximo. Devido à problemas com a identificação de amostras, os dados da Estação \# 1 da Campanha \# 2 não foram utilizados. O total de sedimentos em suspensão foi determinado através do método gravimétrico descrito em Melo et al. (1975).
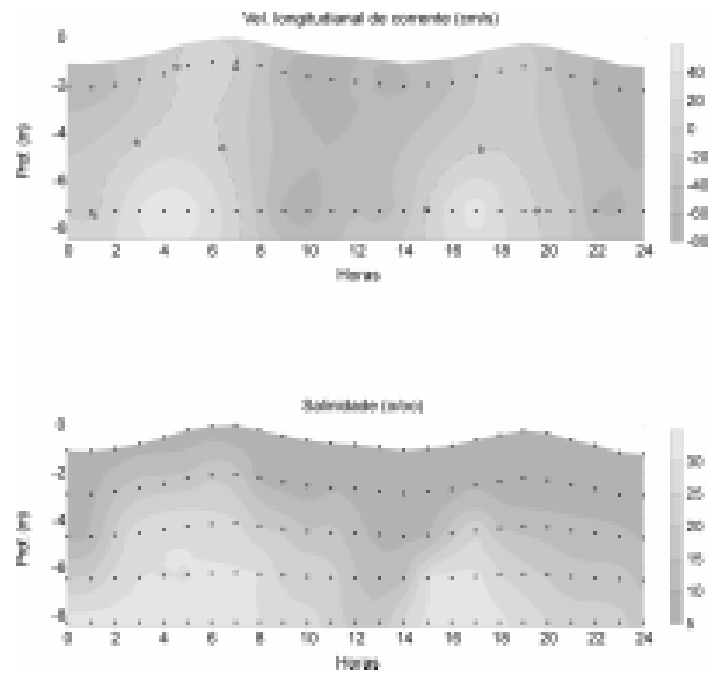

Figura 3: Campo de salinidade e de velocidade de corrente da Campanha \# 1, Estação \# 1.

\section{$V$ - Redução dos dados}

Em função dos diferentes escalas de tempo e espaço utilizadas na obtenção dos dados, todos passaram por um processamento de redução para uniformizar a informação apresentada. Primeiramente foi realizado o ajuste temporal, onde através de interpolação linear se obteve as informações sobre a hora exata, e.g., 14:00, 15:00, ... Os parâmetros que foram coletados a cada metro de coluna de água foram ajustados espacialmente para cinco níveis através de interpolação linear, e ajustados para a coluna de água do instante de coleta. Os dados de corrente foram decompostos em componentes longitudinais e transversais, sendo utilizados somente os primeiros. A velocidade de corrente longitudinal foi definida como positiva para o sentido de corrente entrando no estuário, e negativo no caso contrário. Todos estes procedimentos forma executados através de rotinas para ambiente Matlab ${ }^{\text {TM }}$.
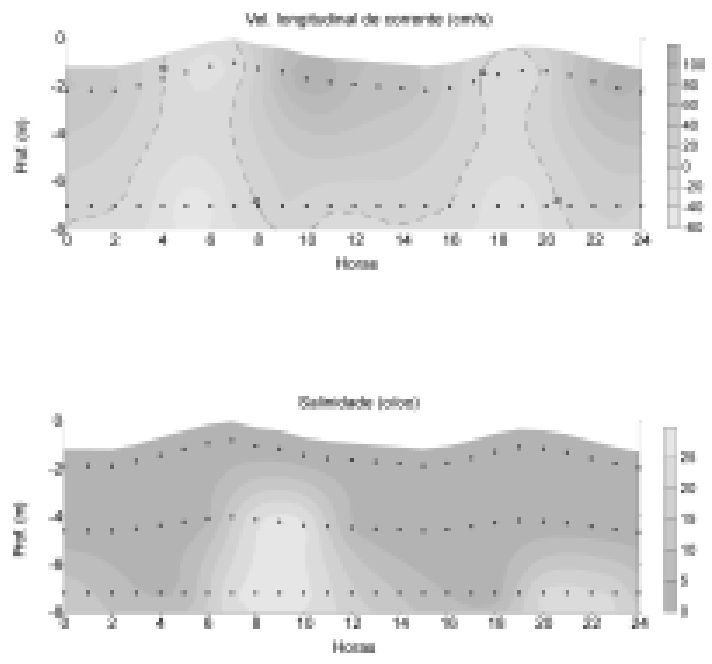

Figura 4: Campo de salinidade e de velocidade de corrente da Campanha \# 1, Estação \# 2. 

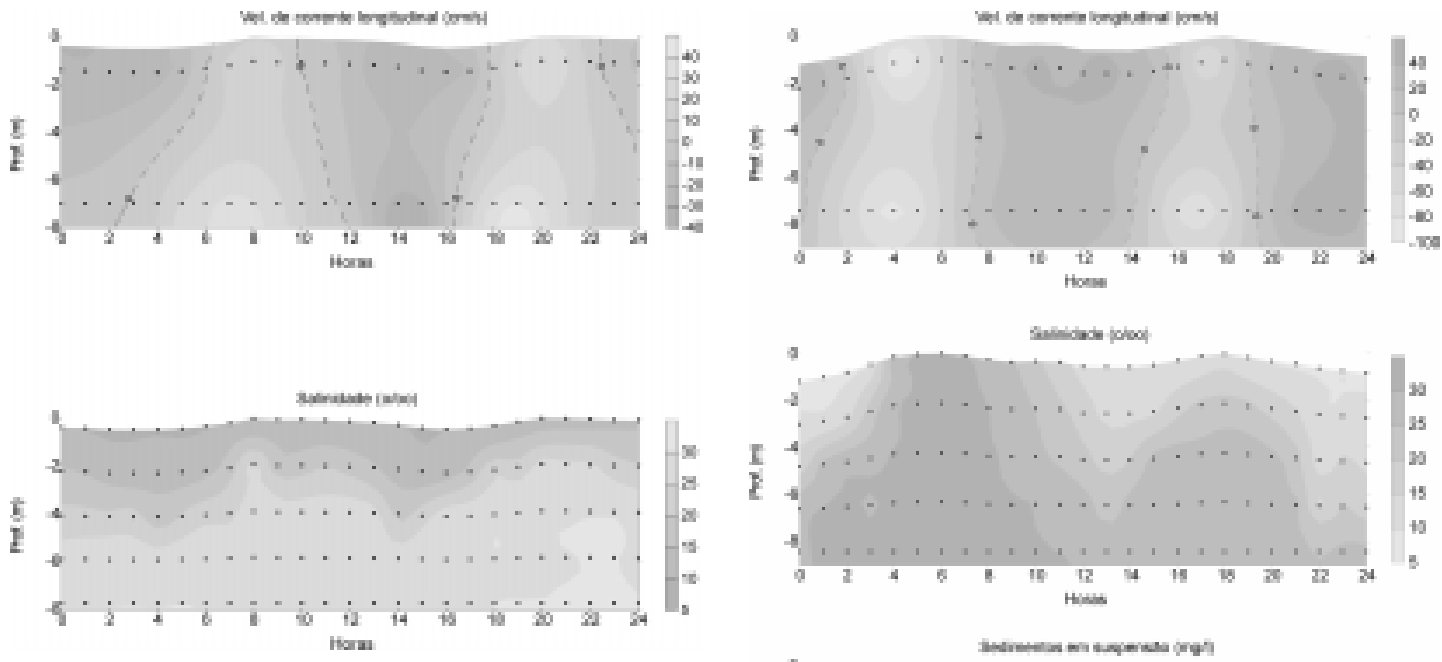

Figura 5: Campo de salinidade e de velocidade de corrente da Campanha \# 2, Estação \# 1.
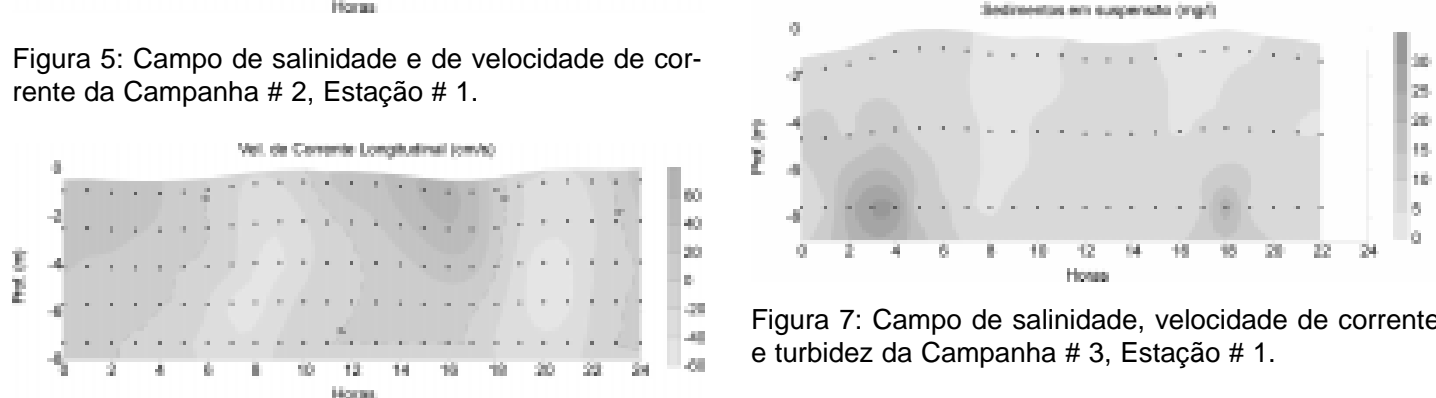

Figura 7: Campo de salinidade, velocidade de corrente e turbidez da Campanha \# 3, Estação \# 1.
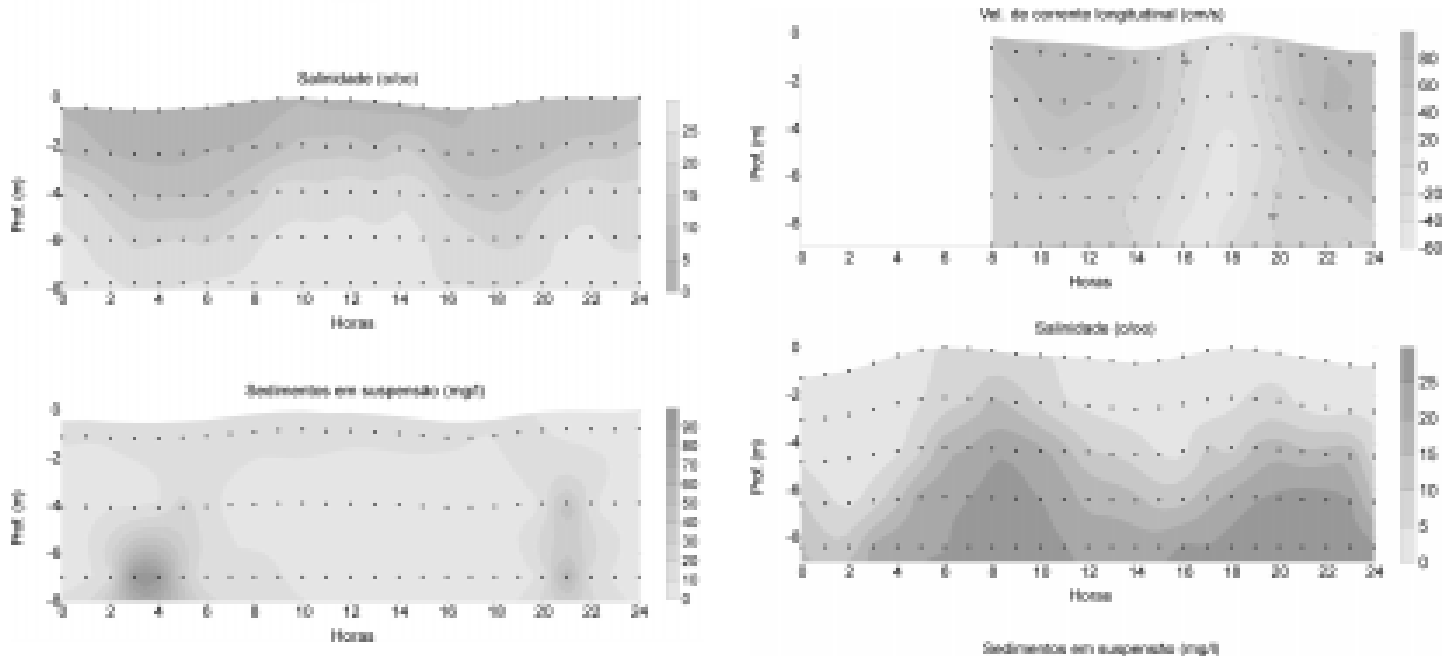

Figura 6: Campo de salinidade, velocidade de corrente e turbidez da Campanha \# 2, Estação \# 2.

Figura 8: Campo de salinidade, velocidade de corrente e turbidez da Campanha \# 3, Estação \# 2.

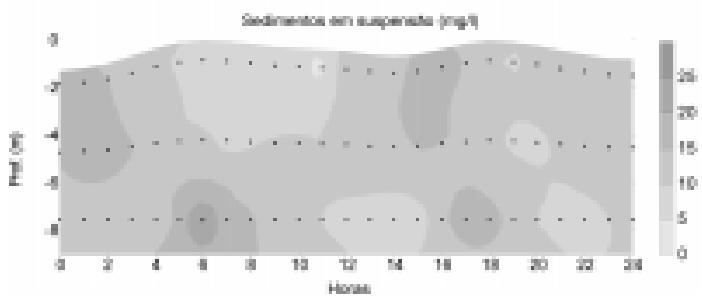


Após a obtenção dos dados reduzidos em tempo e nível de coleta, estes foram interpolados bi-dimensionalmente através do programa Surfer (Golden Software), sendo apresentados como campos de distribuição das variáveis.

\section{RESULTADOS}

Os dados obtidos nas campanhas amostrais são apresentados na sua forma reduzida nos Anexos 1 a 6 . Os dados de nível são apresentados em termos relativos em torno da média, uma vez que as réguas de medições de nível não foram niveladas para um nível de referência comum. As Figuras 3 até 8 apresentam os campos de distribuição temporal dos parâmetros coletados. As Tabelas 1 até 6 apresentam os resultados médios os níveis de superfície, meia água e próximo ao fundo, bem como os valores de variação máxima dos parâmetros amostrados.

\section{AGRADECIMENTOS}

Os autores agradecem o apoio financeiro da Fundação Banco do Brasil; à todos aqueles que participaram das estafantes campanhas de coleta, em especial ao Prof. Antônio H.F. Klein e à Oc. Eliane C. Truccolo pelo apoio na campanha de novembro de 1994 no Rio Itajaí-açu; às Profas. Valéria Bellotto e Kátia Kuroshima nas campanhas de junho de 1995; à Capitania dos Portos de Itajaí pela cessão da embarcação utilizada na Campanha \# 2.

\section{REFERÊNCIAS BIBLIOGRÁFICAS}

Döbereiner, C.E. 1985. Comportamento hidráulico e sedimentológico do estuário do Rio Itajaí-açu - SC. Rio de Janeiro, INPH, Relatório 700/03, 34p.
Melo, U.; Summerhayes, C.P.; \& Toner, L.G. 1975. Metodologia para o estudo do material em suspensão na água do mar. Boletim Técnico da Petrobrás, 18(3/4):115127.

Ponçano, W.L. 1982. Sedimentação atual em área de interesse ao porto de Itajaí - SC. São Paulo, IPT, Relatório 17.502, 56p.

Ponçano, W.L. 1987. Reconhecimento sedimentológico do estuário do Itajaí-açu (SC). Revista Brasileira de Geociências, 17(1):34-41.

Pritchard, D.W. 1955. Estuarine circulation patterns. Proc. Am. Soc. Civ. Eng., 81:No.717.

Schettini, C.A.F. \& Carvalho, J.L.B. 1996. Avaliação preliminar do transporte residual de material em suspensão no estuário do Rio Itajaí-açu, Sc. In: Congresso Brasileiro de Geologia, 39, Salvador, Bahia, Resumos... SBG, pp245-248.

Schettini, C.A.F.; Carvalho, J.L.B.; Jabor, P. 1996. Comparative hydrology and suspended matter distribution of four estuaries in Santa Catarina State Southern Brazil. In: Workshop on Comparative Studies of Temperate Coast Estuaries, Bahía Blanca, Argentina, Proceedings... UNS/IADO/NSF/IAI, pp2932.

Schettini, C.A.F.; Kjerfve, B.; Carvalho, J.L.B. 1997. Dispersão e transporte de sal no estuário do Rio Itajaí-açu em condições de baixa descarga. In: Congresso Latinoamericano Sobre Ciências do Mar, 7, Santos, Resumos Expandidos, ALICMAR/IOUSP, PP424-425.

Schettini, C.A.F. \& Carvalho, J.L.B. (no prelo). Suspended sediment balance in the estuary of Itajaí-açu river during a low discharge period. Anais da Academia Brasileira de Ciências. 
NOTAS TEC. FACIMAR, 2:131-140, 1998

Tabela 1: Resultados médios da Campanha \#1, Estação \# 1.

\begin{tabular}{ccccc}
\hline Nivel & $\overline{\text { Corr }}$ & $\Delta$ Corr & $\overline{\text { Sal }}$ & $\Delta$ Sal \\
\hline S & $-37,9$ & 101,8 & 7,5 & 27,5 \\
M & - & - & 17,2 & 20,6 \\
F & $-10,4$ & 138,6 & 26,8 & 29,0 \\
\hline Media & $-24,2$ & 120,0 & 17,2 & 25,7 \\
\hline
\end{tabular}

Tabela 2: Resultados médios da Campanha \#1, Estação \# 2.

\begin{tabular}{|c|c|c|c|c|}
\hline Nivel & Corr & $\Delta$ Corr & $\overline{\mathrm{Sal}}$ & $\Delta S a l$ \\
\hline S & $-\overline{43,9}$ & 145,6 & 0,9 & 1,5 \\
\hline$M$ & - & - & 5,8 & 26,7 \\
\hline $\mathrm{F}$ & $+4,2$ & 107,6 & 14,1 & 24,7 \\
\hline Média & -19.8 & 126,6 & 10.4 & 17.6 \\
\hline
\end{tabular}

Tabela 3: Resultados médios da Campanha \# 2, Estação \# 1.

\begin{tabular}{ccccc}
\hline Nivel & $\overline{\text { Corr }}$ & $\Delta$ Corr & $\overline{\text { Sal }}$ & $\Delta$ Sal \\
\hline S & $-7,4$ & 66,0 & 10,6 & 5 \\
M & - & - & 25,5 & 15 \\
F & $+8,9$ & 98,0 & 29,6 & 3 \\
\hline Média & $+0,8$ & 82,0 & 21,9 & 7,7 \\
\hline
\end{tabular}

Tabela 4: Resultados médios da Campanha \# 2, Estação \# 2.

\begin{tabular}{ccccccc}
\hline Nivel & $\overline{\text { Corr }}$ & $\Delta$ Cort & $\overline{\text { Sal }}$ & $\Delta$ Sal & $\overline{\text { Turb }}$ & $\Delta$ Turb \\
\hline S & $-14,5$ & 93 & 4,1 & 4 & 11,9 & 10,0 \\
M & $+5,5$ & 88 & 17,7 & 14 & 9,4 & 53,4 \\
F & $+10,1$ & 49 & 25,5 & 2 & 19,8 & 94,8 \\
\hline Média & $+0,4$ & 77,0 & 15,8 & 6,7 & 13,7 & 52,7 \\
\hline
\end{tabular}

Tabela 5: Resultados médios da Campanha \# 3, Estação \# 1.

\begin{tabular}{ccccccc}
\hline Nivel & $\overline{\text { Corr }}$ & $\Delta$ Cort & $\overline{\text { Sal }}$ & $\Delta$ Sal & $\overline{\text { Turb }}$ & $\Delta$ Turb \\
\hline S & $-5,4$ & 152 & 15,6 & 21 & 5,8 & 9,5 \\
M & - & - & 24,6 & 19 & 7,2 & 13,1 \\
F & $+4,8$ & 129 & 28,8 & 7 & 10,1 & 28,1 \\
\hline Média & $-0,3$ & 140 & 23,0 & 15,7 & 7,7 & 16,9 \\
\hline
\end{tabular}

Tabela 6: Resultados médios da Campanha \# 3, Estação \# 2.

\begin{tabular}{ccccccc}
\hline Nivel & $\overline{\text { Corr }}$ & $\Delta$ Cort & $\overline{\text { Sal }}$ & $\Delta$ Sal & $\overline{\text { Turb }}$ & $\Delta$ Turb \\
\hline S & -46 & 112 & 3,4 & 6 & 12,1 & 14,8 \\
M & -15 & 117 & 12,0 & 23 & 12,3 & 8,6 \\
F & +8 & 67 & 24,0 & 18 & 12,8 & 20,7 \\
\hline Média & $-17,7$ & 99 & 13,1 & 15,7 & 12,4 & 14,7 \\
\hline
\end{tabular}


SCHETTINI \& CARVALHO: Hidrodinâmica e distribuição de sólidos em suspensão no estuário do Rio Itajaí-açu.

ANEXO 2: Dados reduzidos da Campanha \# 1, Estação \# 2. Tempo $0=20: 00$ horas do dia 2 de novembro de 1994; O nível oscila em torno do valor médio; Os subscritos indicam o nível fracionado a partir da superfície; $\mathrm{C}$ - corrente longitudinal, em $\mathrm{cm} \cdot \mathrm{s}^{-1} ; \mathrm{S}$ - salinidade em \%.

\begin{tabular}{|c|c|c|c|c|c|c|c|c|}
\hline Tempo & Nivel & $\mathrm{C}_{\mathrm{O} 1}$ & $\mathrm{C}_{23}$ & $S_{000}$ & $S_{2,23}$ & $S_{0 \times 0}$ & $S_{n n}$ & $S_{100}$ \\
\hline 0 & $-0,42$ & $-61,0$ & 0.7 & 7,5 & 8,3 & 9,0 & 20,5 & 32,0 \\
\hline 1 & $-0,43$ & $-48,8$ & $-6,4$ & 7,0 & 8,3 & 9,5 & 20,8 & 32,0 \\
\hline 2 & $-0,32$ & $-42,6$ & 120 & 7,0 & 14,5 & 22,0 & 27,0 & 32,0 \\
\hline 3 & $-0,13$ & $-20,3$ & 42.5 & 8,0 & 18.0 & 28,0 & 30.0 & 32,0 \\
\hline 4 & 0,14 & $-10,7$ & 56.0 & 6,7 & 17,0 & 28,6 & 29.9 & 31,1 \\
\hline 5 & 0.46 & 9.7 & 51.4 & 5.4 & 16.0 & 28.1 & 29.8 & 30,1 \\
\hline 6 & 0,61 & 14,9 & 279 & 8.2 & 19.3 & 26,6 & 30.3 & 30,5 \\
\hline 7 & 0,67 & 4,1 & 7.8 & 4,5 & 18,2 & 26,6 & 30,4 & 30,8 \\
\hline 8 & 0,47 & $-27,2$ & $-29,5$ & 6,0 & 7,9 & 19,4 & 30,5 & 31,0 \\
\hline 9 & 0,22 & $-80,3$ & $-68,6$ & 6,9 & 8,2 & 19,5 & 22,6 & 24,5 \\
\hline 10 & 0,02 & $-74,1$ & $-71,5$ & 4,8 & 6., है & 14,8 & 23,3 & 27,0 \\
\hline 11 & $-0,09$ & $-67,1$ & $-48,3$ & 8,4 & 9.7 & 17,2 & 23,0 & 23,2 \\
\hline 12 & $-0,16$ & $-56,2$ & $-37,5$ & 8,5 & 9.0 & 11,6 & 14.9 & 17,3 \\
\hline 13 & $-0,28$ & $-61,5$ & -48.5 & 7,3 & 8.1 & 8,5 & 13.0 & 15,4 \\
\hline 14 & $-0,40$ & $-62,7$ & $-34,8$ & 7,2 & 7,7 & 8,5 & 12,5 & 26,0 \\
\hline 15 & $-0,31$ & $-54,1$ & 50 & 6,5 & 7,1 & 9,2 & 30,8 & 31,6 \\
\hline 16 & $-0,20$ & $-41,5$ & 29,1 & 5,8 & 7,4 & 17,2 & 31,5 & 31,6 \\
\hline 17 & 0,04 & $-21,9$ & 66.8 & 5,7 & 10,0 & 26,0 & 31,6 & 31,7 \\
\hline 18 & 0,28 & $-4,6$ & 19.4 & 5,2 & 8,4 & 14,2 & 28,0 & 31,1 \\
\hline 19 & 0,44 & $-0,7$ & 10.9 & 5,4 & 5,8 & 13,4 & 22,2 & 28,1 \\
\hline 20 & 0,34 & $-36,6$ & $-9,7$ & 5,6 & 6,3 & 12,6 & 19,3 & 25,0 \\
\hline 21 & 0,05 & $-49,3$ & $-14,6$ & 5,8 & 6.7 & 11,8 & 16.5 & 22,0 \\
\hline 22 & $-0,18$ & $-60,9$ & -65.5 & 6,0 & 7,2 & 11,6 & 17,1 & 22,1 \\
\hline 23 & $-0,48$ & $-83,8$ & $-62,8$ & 6,2 & 7,7 & 11,4 & 17,6 & 22,2 \\
\hline 24 & $-0,57$ & $-68,8$ & $-46,6$ & 6,1 & 6.4 & 9,5 & 13,3 & 16,1 \\
\hline
\end{tabular}

ANEXO 1: Dados reduzidos da Campanha \# 1, Estação \# 1. Tempo $0=20: 00$ horas do dia 2 de novembro de 1994; O nível oscila em torno do valor médio; Os subscritos indicam o nível fracionado a partir da superfície; C - corrente longitudinal, em cm.s ${ }^{-1} ; \mathrm{S}$ - salinidade em \%.

\begin{tabular}{|c|c|c|c|c|c|c|}
\hline Tempe & Nivel & $\mathrm{C}_{21}$ & $\mathrm{C}_{11}$ & $\mathrm{~S}_{200}$ & $S_{0 \mathrm{ng}}$ & $S_{1,00}$ \\
\hline 0 & $=0,44$ & 81,3 & 3.0 & 1,5 & 2 & 13,5 \\
\hline 1 & $=0,44$ & 57,3 & 4,2 & 1 & 2.25 & 10,75 \\
\hline 2 & $=0,46$ & 43,0 & 1,3 & 0,75 & 2.25 & B \\
\hline 3 & $-0,25$ & 24,0 & $-7,1$ & 0,5 & 1.5 & 5.25 \\
\hline 4 & 0,04 & 0,3 & $-29,0$ & 0,25 & 1,5 & 4,5 \\
\hline 5 & 0.32 & -230 & -54.7 & 0.25 & 1.75 & 5 \\
\hline 6 & 0.58 & -275 & $-48,6$ & 0.75 & 3.5 & 14.5 \\
\hline 7 & 0,72 & -180 & $-8,8$ & 1,25 & 14.25 & 25 \\
\hline 8 & 0.48 & 26,1 & -2.6 & 1.25 & 24.5 & 26 \\
\hline 9 & 0,34 & 91,4 & 22,5 & 1,26 & 27.5 & 26,5 \\
\hline 10 & 0,04 & 116,7 & 12,1 & 1,75 & 27 & 26,5 \\
\hline 11 & $-0,11$ & 108,8 & 0,7 & 1,75 & 14.75 & 21,5 \\
\hline 12 & $-0,19$ & 76.2 & 2.5 & 1,25 & 4.25 & 15,5 \\
\hline 13 & -0.27 & 69.9 & 4.8 & 1 & 2.75 & 13 \\
\hline 14 & $-0,35$ & 71,5 & 9.6 & 0,75 & 1,25 & 10 \\
\hline 15 & $-0,44$ & 57,9 & 3,0 & 0,5 & 1 & 7 \\
\hline 16 & $-0,32$ & 31,7 & 1,5 & 0,5 & 0,75 & 5 \\
\hline 17 & $=0,09$ & 6,2 & $-13,5$ & 0,26 & 0.75 & 2,75 \\
\hline 18 & 0,21 & $-13,7$ & $-34,1$ & 0,26 & 1 & 1,75 \\
\hline 19 & 0,40 & -18.3 & $-44,1$ & 0,75 & 1.5 & 12 \\
\hline 20 & 0,35 & 5,7 & $-7,6$ & 0,75 & 1,75 & 22 \\
\hline 21 & 0,18 & 54,9 & 5.8 & 0.5 & 1,75 & 22,5 \\
\hline 22 & $-0,10$ & 94,9 & 22,3 & 0,6 & 1.9 & 21,1 \\
\hline 23 & $-0,35$ & 110,0 & 31,3 & 0,75 & 2 & 19,75 \\
\hline 24 & $-0,55$ & 95,1 & 12,5 & 0,75 & 2 & 12,3 \\
\hline
\end{tabular}


ANEXO 4: Dados reduzidos da Campanha \# 2, Estação \# 2. Tempo $0=12: 00$ horas do dia 6 de junho de 1995; O nível oscila em torno do valor médio; Os subscritos indicam o nível fracionado a partir da superfície; C - corrente longitudinal, em cm.s ${ }^{-1} ; \mathrm{S}$ - salinidade em \%o. T - sedimentos em suspensão, em mg. $\mathrm{I}^{-1}$.

\begin{tabular}{|c|c|c|c|c|c|c|c|c|c|c|c|c|c|c|}
\hline Tempo & Niver & $\mathrm{C}_{913}$ & $C_{0 x}$ & Cene & $\mathrm{C}_{x}=$ & $\mathrm{C}_{0 \times \infty}$ & $\mathrm{Sec}_{\mathrm{e}}$ & $\mathbf{S}_{x 39}$ & $S_{0,00}$ & $50 \pi$ & $\mathbf{S}_{\text {×ce }}$ & $T_{a p}$ & Tos & $T_{E B}$ \\
\hline 0 & $-0,17$ & $-17,1$ & -37.5 & $-23,3$ & $-10,3$ & $-11,5$ & 3.8 & 9,7 & 23,7 & 25.6 & 28,0 & 12,4 & 4,4 & 60 \\
\hline 1 & $-0,15$ & $-27,2$ & -20.5 & $-14,8$ & $-12,9$ & $-8,9$ & 4.8 & 6,8 & 19.9 & 24.7 & 25,6 & 11,2 & 6.4 & $8 . \mathrm{B}$ \\
\hline 2 & -0.23 & $-41,4$ & -320 & $-14,0$ & -6.2 & $-0,8$ & 32 & 4,7 & 14.4 & 22.8 & 25,6 & 11,8 & 8.0 & 15.4 \\
\hline 3 & -0.28 & $-39,4$ & -22.1 & -60 & -0.1 & 1,1 & 2.9 & 3.8 & 10,4 & 17.8 & 24.3 & 16.0 & 6.0 & 69.2 \\
\hline 4 & -0.26 & -24.7 & -17.4 & -1.9 & -0.6 & 0.3 & 28 & 3.0 & 9.7 & 12.5 & 24.0 & 17.8 & 8.8 & 285. 4 \\
\hline 5 & -0.21 & $-1,0$ & -43 & 0.7 & $0, \theta$ & 18.6 & 2.6 & 3,8 & 0,6 & 20,4 & 28,0 & 15,8 & 24.4 & 33,0 \\
\hline 6 & 0,14 & 1,8 & -2.3 & 2,6 & 19,2 & 21.4 & 3,1 & 4,9 & 9.6 & 18,4 & 25,4 & 16,0 & 13.6 & 11,2 \\
\hline 7 & 0,00 & 15,0 & 13.7 & 12,8 & 45,9 & 37,0 & 3,4 & 5,1 & 12,4 & 21,3 & 26,5 & 14,4 & 7,4 & 10,2 \\
\hline 8 & 0,12 & 26,3 & 34,1 & 41,6 & 47,2 & 36.5 & 3.6 & 5,4 & 15,2 & 24,3 & 25,7 & 11,6 & 6,0 & 18,2 \\
\hline 9 & 0.20 & 19,4 & 38.0 & 45,9 & 29.5 & 15.8 & 3.3 & 7,8 & 23,1 & 25.5 & 25,8 & 12,4 & 4,8 & 10,9 \\
\hline 10 & 0.23 & 7,8 & 22.8 & 24,8 & 12,9 & 3,9 & 4.9 & 11,7 & 22.3 & 25.8 & 25,9 & 12.8 & 5.2 & 6.4 \\
\hline 11 & 0.18 & -17.5 & 5.1 & 13.3 & 4.2 & 0.0 & 32 & 11.5 & 21.4 & 25.5 & 25.8 & 11,8 & 4.8 & 62 \\
\hline 12 & 0.17 & $-36,8$ & -49 & 3.0 & 0,6 & 0.0 & 3.5 & 12,8 & 24,4 & 25.7 & 20.0 & 11,0 & 42 & 6.6 \\
\hline 13 & 0,11 & 32,5 & 18.5 & -3.7 & 2,6 & 0,0 & 3.5 & 9,1 & 21,4 & 25.1 & 20,9 & 11,2 & 4,8 & 7.6 \\
\hline 14 & 0.01 & $-50,5$ & .32 .6 & $-12,7$ & $-4,4$ & 0,0 & 3,0 & 15,6 & 23,9 & 25,5 & 26,8 & 11,8 & 5,8 & 6.8 \\
\hline 16 & $-0,11$ & $-66,2$ & -47.6 & $-21,9$ & $-9,2$ & 0,0 & 3.0 & 14,0 & 24,0 & 25,3 & 26,4 & 12,6 & 4,6 & 6.6 \\
\hline 16 & -0.22 & $-66,6$ & -699 & $-30,8$ & $-8,2$ & 0,0 & 3.4 & 6,2 & 16,2 & 23,5 & 24,8 & 12,4 & 3.8 & 5.8 \\
\hline 17 & -0.22 & $-53,9$ & $-51,6$ & -20.7 & $-3,9$ & 0,0 & 5.2 & 6,2 & 13,0 & 21,4 & 25,0 & 10,4 & 7,0 & 4.4 \\
\hline 18 & $-0,12$ & -9.0 & -0.5 & -3.4 & 11.7 & 240 & 5.8 & 7,5 & 12.2 & 18.3 & 23,9 & 10.0 & 9.0 & 4.8 \\
\hline 19 & 0.06 & 18.6 & 19.2 & 29.3 & 34,8 & 322 & 5.2 & 7,0 & 13.0 & 18.0 & 25.0 & 11.2 & 72 & 62 \\
\hline 20 & 0.20 & $23 \pi$ & 28.0 & 57,1 & $\$ 2,3$ & 35.1 & 5.0 & 7,0 & 17.5 & 24.4 & 25,5 & 108 & 7,4 & 10.8 \\
\hline 21 & 0.25 & 28,8 & 35,2 & 43,8 & 46,1 & 33.8 & 5.7 & 10,5 & 212 & 25,1 & 25,6 & 9,0 & 57.2 & 60,2 \\
\hline 22 & 0.24 & 14.2 & 21,8 & 19,8 & 16,6 & 12.9 & 5.5 & 11,6 & 23,5 & 25.6 & 20,0 & 8,2 & 8,2 & 12,4 \\
\hline 23 & 0.22 & $-2,4$ & 6,3 & 5,8 & $-0,1$ & 0,7 & 5,1 & 11,5 & 20,8 & 24,3 & 25,6 & 9,4 & 6.4 & 9,4 \\
\hline 24 & 0,27 & $-33,9$ & $-19,3$ & $-1,2$ & $-0,7$ & 0,0 & 7,2 & 14,8 & 21,9 & 24,7 & 25,8 & 7,8 & 8,4 & 6.4 \\
\hline
\end{tabular}

ANEXO 3: Dados reduzidos da Campanha \# 2, Estação \# 1. Tempo $0=12: 00$ horas do dia 6 de junho de 1995; $O$ nível oscila em torno do valor médio; Os subscritos indicam o nível fracionado a partir da superfície; C - corrente longitudinal, em $\mathrm{cm} . \mathrm{s}^{-1} ; \mathrm{S}$ - salinidade em \%.

\begin{tabular}{|c|c|c|c|c|c|c|c|c|}
\hline Tempo & Nivel & $\mathrm{C}_{21}$ & $\mathrm{C}_{n,}$ & $8_{1 n}$ & S.0 & $\mathrm{S}_{25}$ & Son & $S_{10 x}$ \\
\hline 0 & $=0,13$ & .292 & $-1,4$ & 10,1 & 15.5 & 21,6 & 27.5 & 29,8 \\
\hline 1 & $=0,18$ & -29.6 & $-3,6$ & 9,8 & 14.6 & 21,7 & 27,9 & 29,7 \\
\hline 2 & $=0,23$ & $-26,4$ & $-2,9$ & 9,2 & 12.7 & 20,8 & 28.5 & 29,8 \\
\hline 3 & $-0,24$ & .280 & 4.7 & 9,5 & 11,7 & 23.4 & 291 & 29,9 \\
\hline 4 & $-0,24$ & $-24,1$ & 7.6 & 9,7 & 13.8 & 15,0 & 26.5 & 29,8 \\
\hline 5 & -0.18 & -173 & 27.6 & 9.4 & 11,9 & 20.8 & 294 & 30.0 \\
\hline 6 & -0.09 & -0.9 & 25.7 & 9.7 & 13.5 & 24,1 & 29.1 & 28.7 \\
\hline 7 & 0,04 & 13,5 & 43,2 & 9,8 & 16.3 & 22,5 & 29.2 & 29,4 \\
\hline 8 & 0,24 & 17,4 & 32,4 & $\theta, 9$ & 26.8 & $2 \theta, 3$ & 29.6 & 29,8 \\
\hline 9 & 0,18 & 16,8 & 26,8 & 10,2 & 15,4 & 28,5 & 29.4 & 29,8 \\
\hline 10 & 0,19 & 39 & 9,9 & 10,4 & 17,7 & 27,2 & 28,3 & 28,3 \\
\hline 11 & 0,16 & -60 & 5,1 & 10,5 & 20,0 & 28,0 & 27,3 & 25,7 \\
\hline 12 & 0,13 & -123 & 0.2 & 10,6 & 17.8 & 28,0 & 28.2 & 28,3 \\
\hline 13 & 0,07 & $-27,0$ & $-20,2$ & 10,7 & 15.6 & 27,9 & 29.1 & 29,8 \\
\hline 14 & $-0,01$ & -25.3 & $-42,0$ & 10,1 & 11,5 & 17,0 & 25.4 & 28,4 \\
\hline 15 & $-0,15$ & -22.4 & $-39,6$ & 8,8 & 11,0 & 22,7 & 29.3 & 29,3 \\
\hline 16 & $-0,22$ & $-21,7$ & $-10,3$ & 10,1 & 122 & $28, \theta$ & 29.5 & 29,5 \\
\hline 17 & $=0,17$ & -21.4 & 19.0 & 10,0 & 15.5 & 28,1 & 29.5 & 29,6 \\
\hline 18 & $=0,06$ & 4,8 & 39,0 & 9,9 & 25,3 & 29,7 & 29,9 & 30,0 \\
\hline 19 & 0,11 & 19,9 & 49.8 & 10,2 & 20.2 & 28,4 & 29.9 & 30,0 \\
\hline 20 & 0,26 & 33,6 & 33,9 & 11,4 & 28,0 & 29,6 & 29.9 & 30,0 \\
\hline 21 & 0.25 & 21,7 & 21.2 & 11,3 & 25.7 & 29.6 & 29.9 & 30,1 \\
\hline 2 & 0.22 & 5,6 & 8,8 & 11.9 & 26.6 & 29,8 & 30.0 & 30,1 \\
\hline 23 & 0,17 & -7.8 & 3,8 & 13,2 & 21.6 & $2 \theta, \theta$ & 30.0 & 30,1 \\
\hline 24 & 0,16 & $-11, \theta$ & 0,2 & 13,6 & 202 & $28, \theta$ & 29.8 & 30,1 \\
\hline
\end{tabular}


SCHETTINI \& CARVALHO: Hidrodinâmica e distribuição de sólidos em suspensão no estuário do Rio Itajaí-açu.

ANEXO 5: Dados reduzidos da Campanha \# 3, Estação \# 1. Tempo 0 = 9:00 horas do dia 13 de junho de 1995; O nível oscila em torno do valor médio; Os subscritos indicam o nível fracionado a partir da superfície; $\mathrm{C}$ - corrente longitudinal, em cm.s ${ }^{-1} ; \mathrm{S}$ - salinidade em \%. T - sedimentos em suspensão, em mg.t-1.

\begin{tabular}{|c|c|c|c|c|c|c|c|c|c|c|c|}
\hline Tempo & Nivel & $\mathrm{C}_{01}$ & $\mathrm{C}_{11}$ & $\mathrm{~S}_{20}$ & $S_{034}$ & $S_{0,8}$ & $\mathrm{~S}_{13}$ & $S_{180}$ & $T_{11}$ & $T_{0.5}$ & $T_{29}$ \\
\hline 0 & $=0,77$ & 33.7 & $-11,3$ & 9,40 & 11,30 & 16.40 & 29,10 & 29.50 & 7,4 & 11,1 & 4.8 \\
\hline 1 & $=0,59$ & $-27,9$ & 26,1 & 8,78 & 10,90 & 26,00 & 30,22 & 30,39 & 9,7 & 12.5 & 9,2 \\
\hline 2 & $=0,38$ & $-4,4$ & 45,1 & 8,73 & 13,12 & 29,10 & 30,44 & 30,60 & 8,6 & s. 5 & 22,9 \\
\hline 3 & $-0,04$ & 51,5 & 75,8 & 9,36 & 22,08 & 28,39 & 29,58 & 30,18 & 7,7 & 13.3 & 31,6 \\
\hline 4 & 0,29 & 96,9 & 87.4 & 29,90 & 30,05 & 30,40 & 30,53 & 30,10 & 9,9 & 13.2 & 31,9 \\
\hline 5 & 0.41 & 81.4 & 70.8 & 30.07 & 30.35 & 30.49 & 30.50 & 30.44 & 7.7 & 64 & 9.2 \\
\hline 6 & 0.43 & 43.9 & 37.9 & 30.20 & 30.20 & 30,30 & 30,30 & 30.40 & 9.8 & 80 & 12,1 \\
\hline 7 & 0,34 & 6,4 & 3.3 & 30,40 & 30,30 & 30,30 & 30,30 & 30,30 & 6,3 & 6.2 & 5,7 \\
\hline 8 & 0,17 & -18.0 & $-18,1$ & 28,50 & 30,10 & 30,30 & 30,30 & 30,30 & 0,8 & 4.3 & 3,8 \\
\hline 9 & 0,08 & -34.6 & $-35,8$ & 25,86 & 28,48 & 29,38 & 30,30 & 30,07 & 0,6 & 0.2 & 6,6 \\
\hline 10 & 0,10 & $-46,0$ & $-38,9$ & 21,00 & 24,60 & 26,10 & 30,00 & 30,20 & 0,4 & 8. 2 & 6,9 \\
\hline 11 & 0,05 & -36.1 & $-29,7$ & 16,89 & 21,12 & 24,05 & 27,32 & 29,93 & 6,0 & 5.1 & 5,9 \\
\hline 12 & $-0,15$ & $-40,1$ & $-35,5$ & 13,40 & 18,20 & 22,60 & 29,30 & 29,80 & 5,9 & 6.7 & 6.8 \\
\hline 13 & $-0,16$ & -48.9 & $-28,7$ & 11,40 & 13,10 & 16,70 & 20,40 & 26,80 & 5,7 & 7,0 & 6,5 \\
\hline 14 & $-0,17$ & -33.4 & $-5,8$ & 11.60 & 13.60 & 19,80 & 27,80 & 28.10 & 6.2 & 6.1 & 7,5 \\
\hline 15 & $-0,06$ & $-24,1$ & 26,7 & 11,20 & 13,70 & 27,00 & 28,20 & 28,40 & 4,9 & 6.2 & 8,8 \\
\hline 16 & 0,13 & 14,1 & 64,0 & 10,40 & 18,60 & 27,50 & 28,30 & 28,40 & 4,8 & 4.2 & 5,1 \\
\hline 17 & 0,31 & 66,7 & 75,4 & 10,90 & 21,30 & 26,00 & 27,20 & 26,20 & 4,8 & 5.8 & 8,0 \\
\hline 18 & 0,42 & 42,1 & 52,2 & 14,13 & 22,01 & 27,20 & 27,00 & 26,35 & 4,9 & 5.1 & 27,0 \\
\hline 19 & 0,25 & -0.3 & 11,2 & 9,70 & 21,25 & 27,65 & 28,40 & 28,40 & 4,7 & 80 & 6,7 \\
\hline 20 & 0,10 & -25.8 & $-21,6$ & 9,40 & 18,13 & 25,95 & 28,23 & 28,40 & 5,6 & 90 & 6,7 \\
\hline 21 & $-0,02$ & -36.1 & $-48,7$ & 9.40 & 15.83 & 23,19 & 27,25 & 28,00 & 4,9 & 4.5 & 9,2 \\
\hline 22 & $-0,21$ & -42.5 & $-44,3$ & 9,50 & 9,90 & 10,90 & 13,40 & 23.70 & 5,5 & 50 & 9,2 \\
\hline 23 & $-0,32$ & $-42,4$ & $-45,3$ & 10,10 & 10,70 & 17,00 & 21,38 & 26,90 & - & - & - \\
\hline 24 & $-0,42$ & -37.9 & $-38,8$ & 9,60 & 10,80 & 13,70 & 16,80 & 26.40 & - & - & - \\
\hline
\end{tabular}

ANEXO 6: Dados reduzidos da Campanha \# 3, Estação \# 2. Tempo 0 = 9:00 horas do dia 13 de junho de 1995; O nível oscila em torno do valor médio; Os subscritos indicam o nível fracionado a partir da superfície; $\mathrm{C}$ - corrente longitudinal, em cm. $\mathrm{s}^{-1} ; \mathrm{S}$ - salinidade em \%. T - sedimentos em suspensão, em mg.l-1.

\begin{tabular}{|c|c|c|c|c|c|c|c|c|c|c|c|c|c|c|}
\hline empo & Niver & $\mathrm{C}_{213}$ & $\operatorname{Cos} \times$ & Cene & $\mathrm{C}_{2 n}$ & $\mathrm{Cog}_{000}$ & Sec & $\boldsymbol{S}_{x \geq 3}$ & Somo & $50 \%$ & $\mathbf{S}_{\text {va }}$ & $T_{Q p}$ & Tos & $T_{E S}$ \\
\hline 0 & $-0,89$ & - & - & - & - & - & 2,1 & 2,6 & 4,8 & 11,1 & 15,1 & 17,1 & 14.9 & 14,1 \\
\hline 1 & -0.78 & - & - & - & - & - & 14 & 1,8 & 2,7 & 64 & $11 \mathrm{~B}$ & 152 & 187 & 13,7 \\
\hline 2 & -0.50 & - & - & - & - & - & $a .7$ & 1,4 & 2.3 & 3.8 & 10,9 & $15 \%$ & 18.1 & 13.8 \\
\hline 3 & -0.27 & - & - & - & - & - & 1.2 & 1.4 & 2.2 & 8.0 & 22.0 & 14.6 & 154 & 14.5 \\
\hline 4 & 0.06 & - & - & - & - & - & 28 & 2.8 & 4.1 & 13.3 & 20.2 & 108 & 120 & 113 \\
\hline 5 & 0,30 & . & , & . & . & . & 40 & 4,5 & B.s & 22.5 & 24,2 & 9,6 & 8.9 & 16,1 \\
\hline 6 & 0,38 & * & " & . & * & $=$ & 5.5 & 7,9 & 18,9 & 23.4 & 24,8 & 9,9 & 11,5 & 27,4 \\
\hline 7 & 0,36 & - & - & - & - & - & 6.6 & 8,8 & 19.5 & 25.0 & 25,8 & 8,0 & 8,1 & 14,6 \\
\hline 8 & 0.25 & $-56,4$ & -19.8 & $-10,0$ & $-5,4$ & 0,0 & 7.2 & 16,0 & 24.6 & 28.0 & 26,5 & 6,0 & 9.6 & 16,7 \\
\hline 9 & 0,12 & $-75,6$ & -30.1 & -28.0 & $-13,4$ & 0,0 & 5.8 & 13,2 & 23.8 & 25.8 & 26,7 & 6,7 & 10.5 & 10,8 \\
\hline 10 & $0,0 \theta$ & $-81,5$ & -62.4 & $-38,7$ & $-10,6$ & 0.0 & 5.8 & 8,8 & 21.4 & 250 & 28,1 & 7,5 & 10.8 & 14,7 \\
\hline 11 & 0.00 & -78.3 & -70.8 & -4.0 & -6.3 & 0.0 & 4.3 & 5.4 & 175 & 23.4 & 25.6 & 2.3 & 100 & 92 \\
\hline 12 & $-0,06$ & $-e, 5$ & -57.7 & $-34,4$ & $-3,1$ & $-\theta .6$ & 3.8 & 4.1 & 10.4 & 17.7 & 22,5 & 14.5 & 11,2 & 8.7 \\
\hline 13 & -0.20 & $-63,3$ & $-65,1$ & $-18,4$ & $-0,4$ & $-7,5$ & 3.2 & 3,7 & 9,9 & 18.8 & 22,4 & 14.2 & 140 & 90 \\
\hline 14 & $=0.27$ & 58,3 & .60 .2 & $=11,5$ & 0,9 & 0,0 & 27 & 3,1 & 8,5 & 16.8 & 23,2 & 14,2 & 13.9 & 76 \\
\hline 16 & .0 .20 & -24.8 & -19.0 & 0.0 & 12.2 & 290 & 22 & 2,7 & 4.7 & 126 & 21,8 & 15,7 & 15.4 & 6.7 \\
\hline 16 & 0,02 & $-6,4$ & 3.0 & 15,7 & 28,5 & 46.6 & 1,9 & 2,7 & 3,3 & 13.2 & 26,3 & 16,0 & 14.9 & 14,1 \\
\hline 17 & 0.25 & 21,4 & 31,1 & 35,9 & 46,3 & $5 \mathrm{~S}_{2} 2$ & 2.3 & 2,9 & 12,0 & 18,1 & 25,0 & 14,9 & 14.8 & 17,5 \\
\hline 18 & 0.37 & 298 & 34.8 & 47,4 & 48,2 & 18.7 & 3.2 & 3.5 & 7,1 & 21.3 & 27,1 & 12.9 & 11,8 & 16.3 \\
\hline 19 & 0.31 & 7,4 & 12.7 & 25.2 & 17.8 & 7.6 & 3.8 & 6.0 & 16.4 & 24.5 & 20.6 & 8.1 & 8.0 & 14.0 \\
\hline$\infty$ & 0.17 & -30.1 & -15.5 & 5.8 & -6.8 & $-1,8$ & 3.5 & 7.3 & 183 & 26.1 & 29.0 & 14.6 & 8.4 & 9.7 \\
\hline 21 & -0.03 & -67.5 & -65.8 & $-23,0$ & $-16,2$ & $-3,4$ & 2.6 & 3,8 & 15.5 & 27,3 & 28,5 & 13,4 & 10.6 & 9.3 \\
\hline 22 & -0.20 & 81,3 & 05.6 & 51.3 & -14.8 & $-1,1$ & 24 & 2,8 & 16.8 & 25.6 & 28.2 & 14.3 & 11,9 & $B .9$ \\
\hline 23 & $-0,37$ & $-76,3$ & $-78,4$ & $-60,8$ & -19,3 & 2,9 & 2.6 & 4,6 & 19,9 & 25,0 & 28,6 & 14,6 & 14.3 & 10,0 \\
\hline 24 & $-0,39$ & $-76,9$ & -72.4 & $-68,0$ & $-47,7$ & 1,5 & 3.0 & 3,5 & 6,4 & 13.8 & 25,8 & 13,5 & 120 & 10,9 \\
\hline
\end{tabular}

